Revue des patrimoines

4 | 2004

Les réseaux de la villégiature

\title{
Hossegor (Landes), une station au cœur des réseaux de villégiature
}

\section{Claude Laroche}

\section{OpenEdition}

1 Journals

Édition électronique

URL : http://journals.openedition.org/insitu/2102

DOI : 10.4000/insitu.2102

ISSN : 1630-7305

Éditeur

Ministère de la Culture

Référence électronique

Claude Laroche, « Hossegor (Landes), une station au cœur des réseaux de villégiature », In Situ [En ligne], 4 | 2004, mis en ligne le 19 avril 2012, consulté le 08 septembre 2020. URL : http:// journals.openedition.org/insitu/2102 ; DOI : https://doi.org/10.4000/insitu.2102

Ce document a été généré automatiquement le 8 septembre 2020

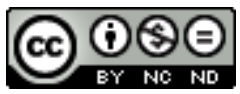

In Situ Revues des patrimoines est mis à disposition selon les termes de la licence Creative Commons Attribution - Pas d'Utilisation Commerciale - Pas de Modification 4.0 International. 


\title{
Hossegor (Landes), une station au cœur des réseaux de villégiature
}

\author{
Claude Laroche
}

Figure 1

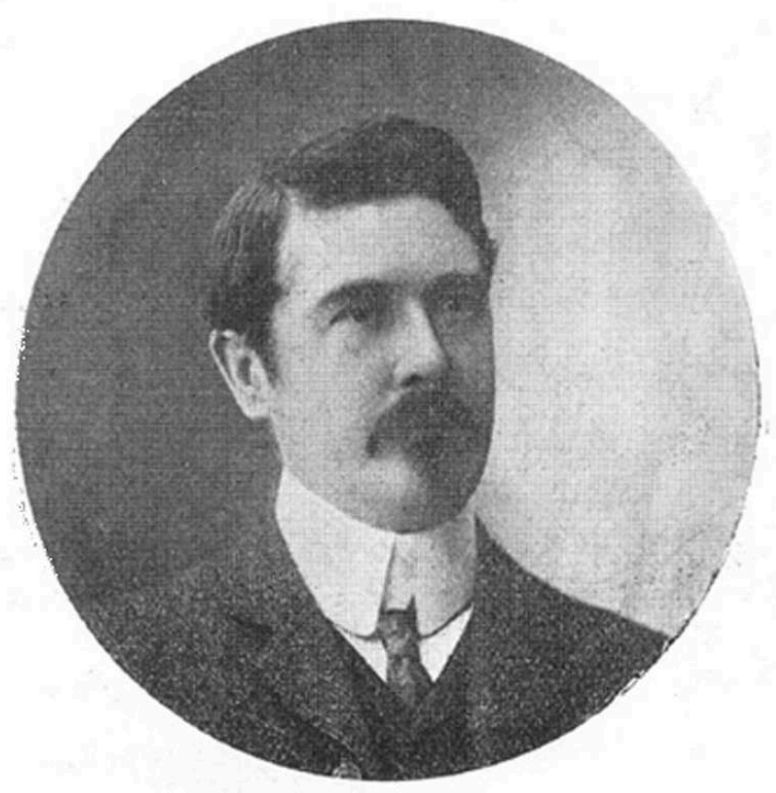

J. H. Rosny jeune.

J.-H. Rosny jeune (Justin Boex, dit), extrait de Hossegor : son lac - sa forêt - sa mer sauvage ; monographie éditée par la revue PYRÉNÉES-OCÉAN

Repro Inv. C. Laroche @ C C. Laroche. Droits réservés

1 Sans entrer dans les détails, commençons par brosser un très bref historique de la station d'Hossegor ${ }^{1}$. Faisant partie de la commune de Soorts-Hossegor, elle est localisée 
dans le département des Landes, au sud de la côte landaise, à vingt-cinq kilomètres au nord de Biarritz. Elle se déploie en grande partie autour d'un lac allongé parallèle au rivage maritime - occupant en fait un ancien bras de l'Adour - lac mis en communication avec l'Océan via un canal à la fin du XIX ${ }^{e}$ siècle. Au tout début du XX siècle, c'est un lieu agreste, d'économie sylvicole et pastorale ; en l'absence de pont, un passeur aide à franchir le canal. Le lieu est découvert et révélé par des hommes de lettres. C'est tout d'abord Justin Boex dit J.-H. Rosny jeune (fig. nº 1) (1859-1948, académicien Goncourt et frère de l'auteur de La Guerre du feu, J.-H. Rosny aîné), le préfacier de La Côte d'Argent de Maurice Martin. On voit donc commencer à poindre la notion de réseau de même que celle de l'ambivalence des personnages. Rosny zélateur et apologue de la vitesse est aussi un contemplatif. Rosny jeune vient sur le site en 1900 par l'entremise du journaliste Mathias Morhardt, Landais d'adoption : Bernard Toulier a démontré le grand rôle des médias dans ces questions de réseaux, rôle que l'on verra à Hossegor à tous les moments-clefs de l'histoire de la station'2 . A Hossegor, Rosny jeune s'installe dans la maison du passeur - tout un symbole, car il s'agit bien là du passage d'un monde à l'autre, de l'image du passage de témoin qui s'attache à la notion de réseau. Mais il quittera Hossegor en 1921, après que le lieu eut largement inspiré son œuvre.

Figure 2

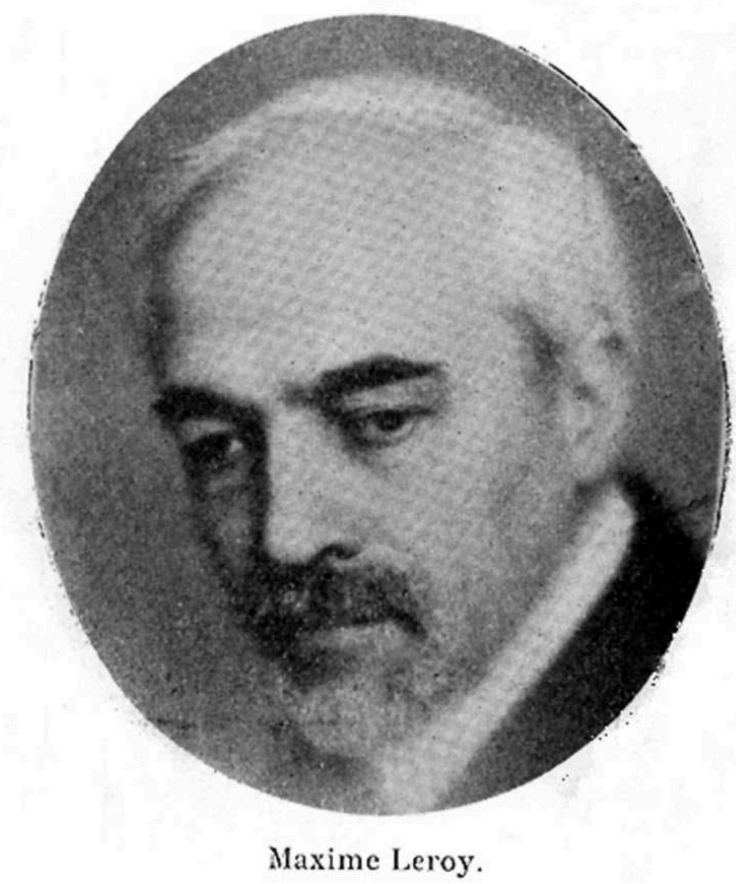

Maxime Leroy, extrait de Hossegor : son lac - sa forêt - sa mer sauvage ; monographie éditée par la revUe PYRÉNÉES-OCÉAN

Repro Inv. Claude Laroche. (C) C. Laroche. Droits réservés

2 En 1903, un autre grand acteur de cette invention du site se joint à Rosny: Maxime Leroy (1873-1957) (fig. $\mathbf{n}^{\circ}$ 2). Magistrat, mais aussi et surtout auteur d'ouvrages dans le domaine de la philosophie, de la sociologie, de l'économie, du droit et de l'histoire des idées. Socialiste, saint-simonien - mais que l'on pourrait dire au second degré, en ce 
sens qu'il est avant tout un historien du saint-simonisme -, Maxime Leroy est également un fervent militant régionaliste ${ }^{3}$.

Figure 3

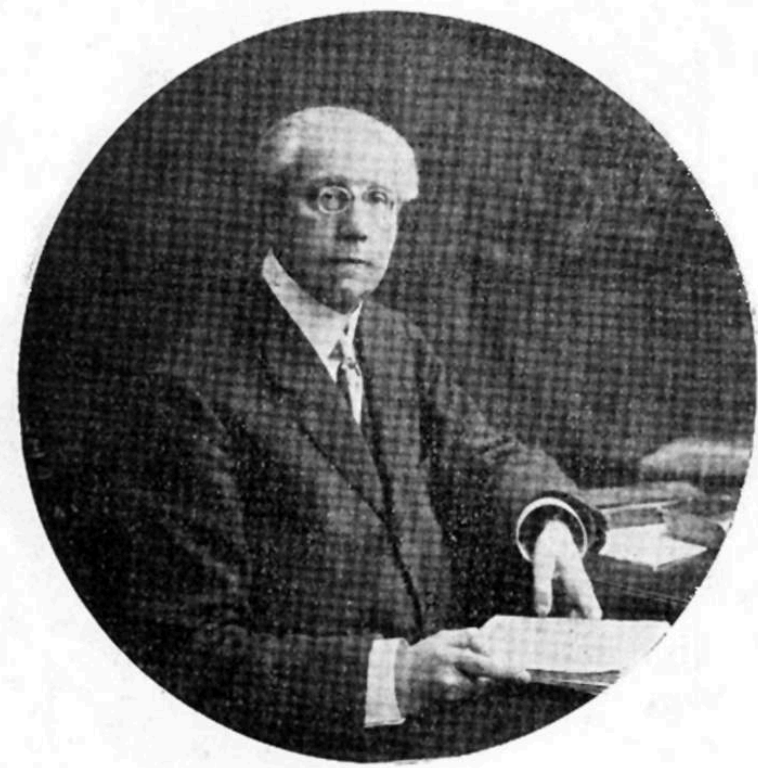

Paul Margueritte.

Paul Margueritte, extrait de Hossegor : son lac - sa forêt - sa mer sauvage ; monographie éditée par la revUe PYRÉNÉES-OCÉAN

Repro Inv. Claude Laroche. (C) C. Laroche. Droits réservés

On peut aussi parler d'un troisième littérateur, Paul Margueritte (né en 1860 et mort à Hossegor en 1918), également académicien Goncourt, alors presque aussi célèbre que son frère Victor (fig. $\mathbf{n}^{\circ}$ 3). Il ne s'installe à Hossegor qu'en 1910. Ces personnalités, ainsi que quelques autres, forment un petit groupe, une "petite société enjouée, amatrice de charades, de déguisements et de promenades sur le lac " ${ }^{4}$, se livrant à de longues discussions, à des repas largement mythifiés au cours desquels ils ne se privent pas de refaire le monde. Ils agrègent au cours des années d'autres hommes de lettres, certains célèbres, comme Gabriele d'Annunzio. Bien que ses membres soient de nature fort disparate, le petit groupe pose volontiers à la communauté phalanstérienne.

Après la contemplation, l'action. Arrive donc, si l'on reprend la désormais célèbre distinction formulée par Dominique Rouillard ${ }^{5}$, le fondateur-promoteur. A Hossegor, il a pour nom Alfred Eluère (1893-1985). En tandem avec un financier, Aimé MeunierGodin et en s'appuyant sur les sociétés immobilières qu'ils ont fondées (et dont les raisons sociales changent au fur et à mesure des nécessaires augmentations de capital), Eluère obtient en 1923 la concession des terrains boisés municipaux moyennant des engagements, notamment en termes de voirie. Les lotissements commencent, tout d'abord sur deux secteurs permettant de tester la faisabilité de l'opération, puis progressivement sur d'autres zones, permises par de nouvelles acquisitions foncières ou par de nouvelles concessions. Les constructions s'élèvent, notamment, outre les villas, le front de mer à partir de 1928, le Sporting-Casino (1927-1928 et 1930-1931), le 
golf (1930). De grands projets sont conçus, mais la crise oblige au recentrage des opérations. En même temps - prévoyait-il une nouvelle donne qui verrait les initiatives publiques venir au premier plan et relayer les initiatives privées défaillantes? - Eluère se retire des sociétés immobilières en 1931 et est élu maire de Soorts-Hossegor en 1935.

Voilà donc à très grands traits l'histoire des débuts de la station, une histoire qui, si on la regarde d'un peu plus près, peut tout à fait être décodée grâce à cette notion de réseau que nous essayons de cerner ces jours-ci, être lue au travers des réseaux de connaissance, des réseaux d'influence, des réseaux de pouvoir. Comme si l'histoire que l'on peut retracer à l'aide des archives, une histoire factuelle un peu sèche et dont on ne comprend pas toujours les enchaînements, était doublée par une autre histoire, souterraine celle-ci et beaucoup plus difficile à mettre au jour - l'histoire de ce qui se trame dans les salles de jeux ou dans les potinières. Cette histoire-là, c'est précisément celle des réseaux. Des réseaux d'hommes : on voit passer des noms, que l'on note tout d'abord sans réagir et puis un jour, la lumière se fait. Cet Untel, c'est bien le même que l'on a rencontré çà et là, qui est président de tel organisme et qui a séjourné à Hossegor à telle date... Ce n'est qu'à partir de là que l'on peut prétendre commencer à comprendre la réalité de certaines choses.

\section{Les premiers réseaux}

En fait, cette notion de réseau imprègne Hossegor dès le début : dès l'invention du lieu. En décrivant la colonie d'écrivains avant tout comme une colonie contemplative, je n'ai fait que suivre le mythe officiel, la légende dorée du lieu. Mais qu'en est-il au juste ? On a vu que Rosny jeune, le sage des bords du lac, était aussi le préfacier de l'ouvrage de Maurice Martin dans une apologie de la vitesse où, quand même, l'automobiliste naissant pourrait bien bousculer quelque peu l'immémorial berger landais juché sur ses échasses! Quant à ces hommes qui, dans le cadre de ce paysage pastoral, jouent en quelque sorte aux bons sauvages, ils ne sont en fait à Hossegor que quelques mois par an. En dehors de cela, ils sont à Paris, où ils publient, ont leurs éditeurs, siègent aux académies, fréquentent les salons, dînent en ville. Ils connaissent donc intimement beaucoup de journalistes. Ils se sont bien rendu compte, déjà, que cette médiatisation journalistique est le seul moyen de faire connaître leur travail. C'est ainsi qu'ils vont se livrer à une première promotion du site, car chez eux, on l'aura compris, la contemplation et l'action, loin de s'exclure ou de se succéder, se mêlent au contraire très intimement.

7 Il faut bien dire qu'ici, le scénario est un peu plus compliqué, un peu plus ambivalent que le schéma habituel, la scansion traditionnelle mise en lumière par Dominique Rouillard pour les stations normandes, c'est-à-dire le scénario de la fuite de l'artiste : les uns faisant fuir les autres, le grand nombre faisant fuir l'élite. Certes Rosny jeune quitte Hossegor en 1921 : il pressent que le destin va basculer et que sa tranquillité ne sera préservée qu'au prix d'un nouvel exil (en l'occurrence la Bretagne, l'île de Bréhat : nouvelle célébration, nouvel acte de dénomination, de baptême - en écho au baptême de la côte d'Argent - l'île de Bréhat deviendra sous sa plume l'Ile des Fleurs... et ce sera reparti !). Rosny quitte donc Hossegor, mais Leroy reste et participe très activement à la promotion de la nouvelle station. Et si Rosny délaisse Hossegor, il n'a pas peu contribué au nouveau destin du lieu, notamment par la retentissante affaire du courant d'Huchet. De quoi s'agit-il ? 
Nous sommes en mai 1911 et, dans l'esprit des expéditions de 1905 qui voient le baptême de la côte d'Argent ${ }^{6}$, est organisée, sur deux jours, une excursion qui pour l'essentiel part de Bordeaux et rejoint le lac de Léon, lac landais dont l'exutoire vers l'Océan est cette rivière littorale, ce fameux " courant d'Huchet ", dont la descente en barque au travers d'épaisses frondaisons est en effet une belle expérience. Cette expédition est en fait un véritable reportage qui associe à Rosny de nombreux journalistes: Maurice Martin, bien sûr, l'état-major de La Petite Gironde (dont son dirigeant, Marcel Gounouilhou, qui fera construire sa maison des environs de Bordeaux par le célèbre architecte Charles Siclis), un photographe de L'Illustration, l'organe commanditaire qui recevra le premier un reportage de l'expédition de la plume de Rosny lui-même (les propriétaires du célèbre journal sont des Landais, les Bachet, originaires de Soustons, juste à côté d'Hossegor - toujours les réseaux...), enfin des littérateurs : Rosny et Margueritte, Charles Derennes et le célèbre d'Annunzio, alors en villégiature au Moulleau, près d'Arcachon.

Descente du courant, arrivée à l'Océan, repas plantureux... Le maire de Moliets offre son vin de sable, les métaphores fusent - on n'en est guère avare à cette époque. Puis Rosny amène d'Annunzio à Hossegor pour lui faire découvrir le lac. Et, de fait, Gabriele d'Annunzio n'aura par la suite de cesse de célébrer cet étang.

Tout cela donne lieu à des articles. Rosny se lance donc le premier dans L'Illustration du 22 juillet de cette même année 1911. L'article s'intitule "Une contrée vierge en France », commence par ces mots : «Il existe, en France, une région presque ignorée de la majorité des Français» et s'achève par un vibrant et lyrique hommage au lac d'Hossegor. Paul Margueritte, quant à lui, relate l'affaire dans Excelsior, Maurice Martin dans la revue mensuelle du Touring Club de France, Charles Derennes de nouveau dans L'Illustration, mais beaucoup plus tard, en 1928, comme une sorte de piqûre de rappel alors que la station d'Hossegor est désormais lancée. Bref, il s'agit là d'un vrai événement journalistique, en ce sens qu'il est quelque peu fabriqué en vue de la promotion d'un lieu et non pas seulement pour faire partager un coup de cœur esthétique et émotionnel pour ce lieu.

11 Réseau de promotion, donc, mais aussi réseau de protection. Car il s'agit de préserver l'endroit, non pas du tourisme, bien au contraire, mais des atteintes qui pourraient en altérer l'esprit et en compromettre l'avenir. C'est ainsi que l'on saura le moment venu contrer des projets de déboisement, des projets d'implantation d'un champ de tir, que l'on saura éloigner la ligne de chemin de fer afin de préserver les terrains susceptibles d'être lotis. C'est là qu'intervient une association, la Société des amis du lac, avec notamment, outre Rosny, Leroy et Martin, l'influent Paul Grunebaum-Ballin, conseiller d'état et ancien bras droit d'Aristide Briand : une association, mais aussi tout le réseau d'influences qu'elle engendre, permettant de faire jouer à bon escient quelques personnalités haut placées. 


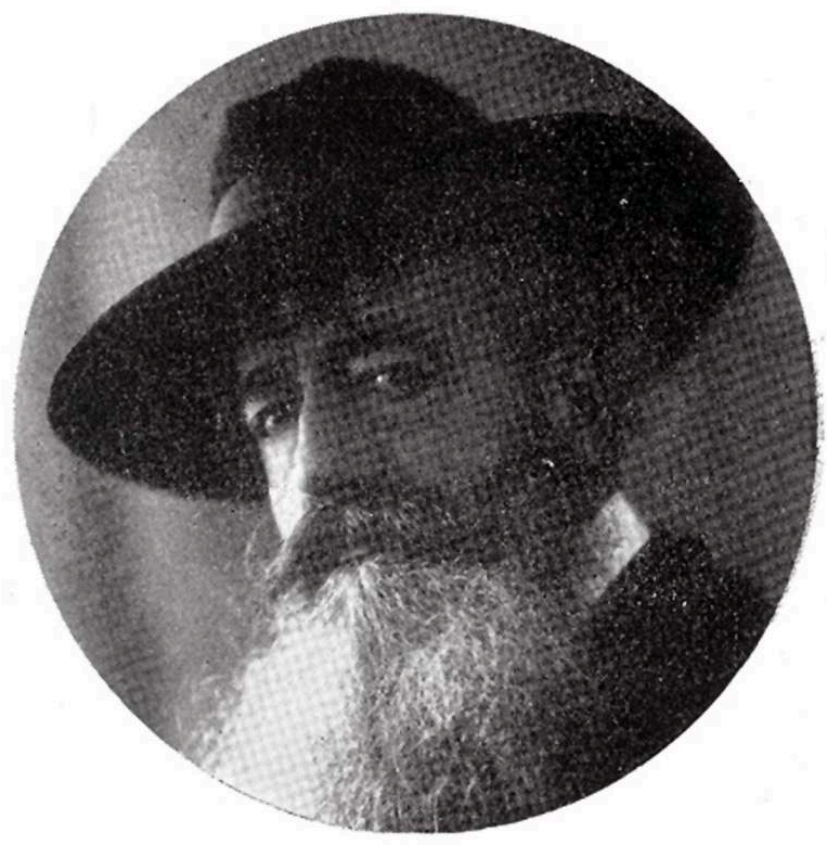

Maurice Martin.

Maurice Martin, extrait de Hossegor : son lac - sa forêt - sa mer sauvage ; monographie éditée par la revUe PYRÉNÉES-OCÉAN

Repro Inv. Claude Laroche. (c) C. Laroche. Droits réservés

12 Il faut bien sûr évoquer un peu plus longuement Maurice Martin (1861-1941), homme de réseau s'il en est (fig. $\mathbf{n}^{\circ}$ 4). Rédacteur sportif à La Petite Gironde, personnage haut en couleur avec sa longue barbe et son béret, personnage important du Touring club de France, mais aussi infatigable vélocipédiste (apte à s'attaquer aux 700 kilomètres de la course Paris-Bordeaux, capable de parcourir à bicyclette la France et l'Europe et ce bien sûr sans dérailleur ni adjuvant...). Maurice Martin fait d'ailleurs la connaissance d'Hossegor à la suite de l'un de ces périples: «Ce fut pour moi, raconte-t-il, une révélation et un prodigieux enchantement. J'arrivais à vélocipède d'un long voyage à travers la Suisse, le nord de l'Italie et tout le Midi français. » Le personnage a d'ailleurs bien failli, pour Hossegor, revêtir la tunique du fondateur-promoteur. Les épisodes sont peu explicites (faisant partie là encore de cette seconde histoire, un peu souterraine, dont on parlait tout à l'heure) et sont encore à documenter si tant est que ce soit possible. Tout d'abord, en 1906, un an après le fameux épisode du baptême de la côte d'Argent, un contrat de concession lie la municipalité de Soorts-Hossegor et la société Vigneau-Darracq. En effet, Vigneau n'est autre que l'ingénieur du boulevard en résine dont Maurice Martin était le co-promoteur. Quant à Darracq, ce n'est personne d'autre qu'Alexandre Darracq, associé également à ce projet de boulevard, président de la Chambre syndicale du cycle et de l'automobile, personne d'autre que le constructeur automobile évoqué par Paul Smith à propos de Deauville (le réseau se resserre humainement, mais s'élargit géographiquement!). Forcément, derrière cette concession - qui cette fois tournera court - et même si son nom ne semble pas apparaître directement, on ne peut pas ne pas voir l'implication de Maurice Martin. 
Ensuite, en 1921, Maurice Martin est partie prenante de la Société d'études des terrains d'Hossegor, qui préfigure la société qui réussira la création de la station, la Société immobilière et artistique d'Hossegor. Celle-ci intervient en 1923 : ce lancement sera enfin le bon; Maurice Martin ne figure pas dans le montage immobilier définitif, pourtant c'est probablement par lui, principalement, qu'intervient le promoteurfondateur que l'histoire retiendra : Alfred Eluère.

\section{Alfred Eluère, l'homme-réseau}

Figure 5

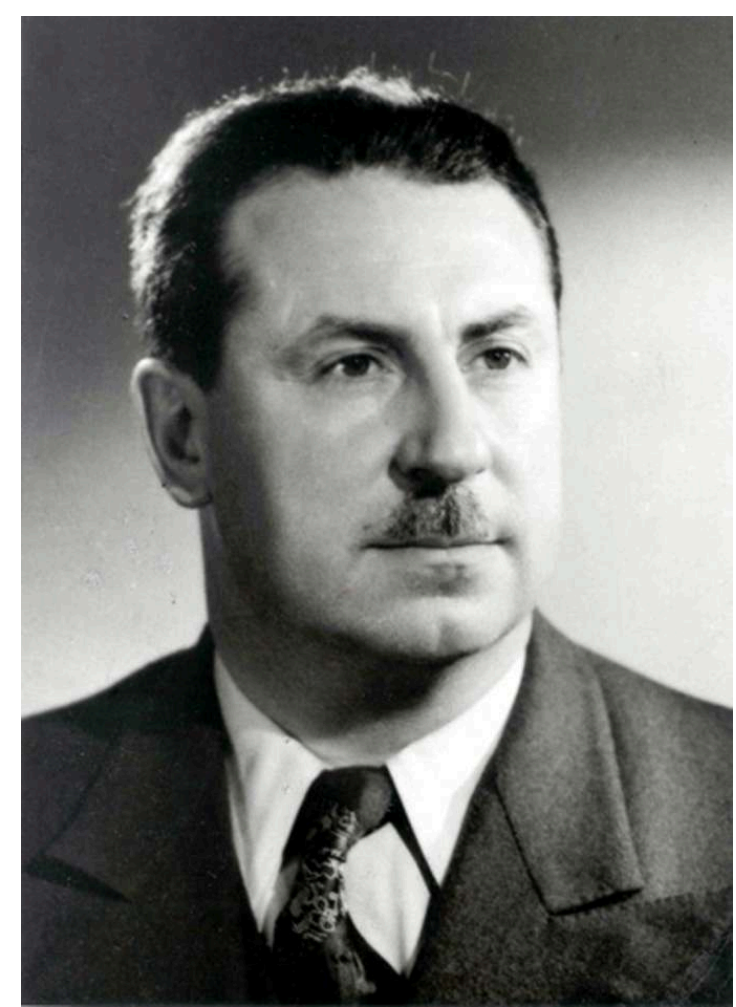

Alfred Eluère au moment du lancement de la station, archives communales de Soorts-Hossegor

Repro Inv. B. Chabot, M. Dubau. (C) B. Chabot, M. Dubau. Droits réservés

14 S'il est un personnage intéressant, touchant au thème abordé ces jours-ci, c'est bien Alfred Eluère - (fig. $\mathbf{n}^{\circ}$ ) ) au même titre d'ailleurs que quelqu'un comme Henry Martinet, le fondateur-promoteur d'Hendaye. Comme celles de Martinet d'ailleurs, les activités d'Eluère recourent à plusieurs domaines, en l'occurrence, le sport, la politique et les affaires. Or, s'il est des domaines où les réseaux ont de l'importance, où même, pourrait-on dire, ils sont primordiaux, ce sont bien ceux-ci: des réseaux qui en l'occurrence interagissent, s'interpénètrent... Eluère, c'est par excellence l'hommeréseau.

Détaillons : en ce qui concerne le sport, Eluère pratique plusieurs disciplines, mais principalement le rugby. Il est international en 1917, plus tard membre du Conseil de la Fédération française de rugby, instance qu'il présidera ensuite de 1942 à 1952. Il sera également président du Comité national des sports de 1947 à 1966. A l'occasion de ces activités, le rugbyman est amené à rencontrer un jeune entrepreneur de Dax, Abel 
Guichemerre (qu'il ne se privera pas de faire travailler par la suite à Hossegor) lui aussi international de 1920 à 1923. Eluère contracte un premier mariage en 1919. Début 1920, le couple effectuant son voyage de noces dans le Sud-Ouest, Eluère va tout naturellement rendre visite à Dax à son ami Guichemerre, lequel l'emmène à Hossegor : c'est donc par le réseau sportif qu'Eluère entre en contact avec le site. Il sera toutefois encore trop tôt et il faudra attendre 1922 pour qu'Eluère, probablement approché par Maurice Martin, se décide à entreprendre. C'est qu'entre-temps, il aura rejoint Aimé Meunier-Godin au sein de la Compagnie d'entreprises immobilières. Ensemble ils réalisent un premier lotissement au Rayol dans le Var et sont à l'affût d'opérations à entreprendre. Puisqu'il est question du voyage de noces d'Eluère, profitons-en pour évoquer un autre réseau : le réseau familial. Les beaux-parents de notre promoteur sont des gens fortunés, ce qui pourra faciliter les choses. De plus, Eluère fera jouer à plein ce réseau familial au moment du démarrage de la Société immobilière et artistique d'Hossegor. Plusieurs de ses frères - dont un juriste - seront mis à contribution et Eluère délèguera sur place son père, Louis, alors âgé de cinquante-huit ans : sa rondeur et sa bonhomie séduiront au point que, localement, Louis Eluère sera affectueusement surnommé « Dieu le père ».

Deuxième domaine : la politique. Inutile d'insister sur le fait qu'il s'agit là d'un lieu de réseaux par excellence. D'autant qu'en l'occurrence Eluère appartient au mouvement politique peut-être le plus travaillé par les réseaux : le radicalisme - appartenance politique doublée d'une appartenance maçonnique. Inutile d'insister non plus s'agissant du troisième domaine, les affaires. Disons simplement qu'Eluère dirigera un temps une société d'électroménager incluant des magasins, notamment à Bayonne, la société Propria, qui s'occupe aussi un peu d'immobilier et dont la raison sociale fournira le titre d'une publication périodique (Propria, revue illustrée pour le Sud-Ouest) qui, en abritant des articles publicitaires et en traitant de la vie mondaine à Hossegor sera le support privilégié de la promotion de la station. On voit ainsi que peu importe le support: le réseau prime l'objet, pourrait-on dire. Peu importe que l'électroménager paraisse bien éloigné de la promotion immobilière et du tourisme, on adoptera le même nom.

Eluère homme-réseau ? Rien ne le montre peut-être mieux que le nom du club privé avec restaurant qu'il fonde à Paris - la ville où lui aussi a toutes ses relations - rue Saint-Augustin et où se nouent bien des intrigues : Le Club des clubs. Déjà, avec ce nom, Eluère affiche clairement l'ambition de créer une tête de réseau. Eluère homme-réseau : il y a chez lui un tel mélange des genres, les choses s'interpénètrent de telle façon que l'on pourrait voir en lui le degré ultime du réseau : un « auto-réseau » en quelque sorte. Un exemple : quand la loi crée, en 1935, les groupements régionaux d'urbanisme, dans le but d'étendre le champ d'application de la loi Cornudet à de plus vastes ensembles, un "groupement régional d'urbanisme des lacs landais et du bas Adour ", intégrant Hossegor, est créé en octobre 1936 dans le cadre de cette loi. Or Eluère est non seulement auteur du plan d'aménagement, mais il essaie en même temps de s'associer à parts égales avec un groupe financier en vue de la réalisation matérielle des opérations prévues à ce programme... Un jour, à Hossegor, voulant faire comprendre à la fois l'esprit d'entreprise d'Eluère et le fait que nous avions affaire à une époque de visionnaires, où le mélange des genres permettait toutes les audaces et que cette époque était bel et bien révolue, je suggérai que s'il opérait de la même façon qu'à l'époque, Eluère serait aujourd'hui mis en examen pour ingérence. J'ai vu alors dans l'assistance opiner d'un air entendu les anciens, ceux qui avait connu et pratiqué le 
bonhomme, silencieuse approbation qui m'assurait que j'avais vu juste. En même temps, je provoquai la fureur de sa veuve, qui avait pris la formule au premier degré et n'avait pas compris que mon propos était en fait empreint d'admiration.

\section{Réseau topographique}

Dans ce balayage de ce que la notion de réseau peut recouvrir, appliquée à la station d'Hossegor, je ne m'étendrai pas sur une acception que j'ai souvent développée : cette notion de nomadisme à l'échelle de l'ensemble de la côte basque et sud landaise, à l'échelle du dispositif qu'elle représente dans son entier, échiquier dans lequel chaque station se spécialise en une sorte de mélange de complémentarité et de concurrence : pour Hossegor, la spécialisation sera matérialisée par le slogan publicitaire, « la station des sports élégants ». Comme support principal de ce nomadisme balnéaire, on pourrait d'ailleurs trouver les publications périodiques dont nous avons parlé plus haut : La côte basque et justement Propria, dont les chroniques mondaines fourmillent d'information sur la présence de telle personnalité à tel endroit ou sur l'occurrence de tel événement : sur tout ce qui peut guider le villégiateur dans sa divagation côtière.

\section{Réseaux de promotion}

Dès le début de l'opération, les promoteurs d'Hossegor utilisent de façon intense et intelligente les différents moyens de promotion à leur disposition. Ils sollicitent la publicité classique - brochures, pages dans la presse - mais utilisent aussi un réseau d'influences qu'ils savent parfaitement faire jouer. Comme le souligne Jean-Claude Drouin, «il est évident que des liens - directs ou indirects - ont existé entre la propagande littéraire et la mise en exploitation des terrains et des maisons ${ }^{7}$. De fait, le talent de plume et le poids personnel d'un littérateur et penseur tel que Maxime Leroy sont très fréquemment mis à contribution. La liste est longue des textes que ce dernier donne, en témoin et acteur de l'« école d'Hossegor ", c'est-à-dire du groupe d'écrivains du début du siècle, en caution intellectuelle en quelque sorte, pour les différentes brochures produites pour la promotion d'Hossegor et dans les numéros spéciaux de revues consacrés en tout ou en partie à la station. Maxime Leroy sait aussi utiliser à des fins publicitaires la gloire d'un des plus illustres visiteurs du site, Gabriele d'Annunzio, qui lui envoie, en 1924, un télégramme s'achevant par une phrase restée célèbre: "Sur le lac de Garde, flotte aujourd'hui l'enchantement d'Hossegor ", une phrase qui sera systématiquement reprise, par Leroy et par d'autres, en exergue de la plupart de leurs textes sur la cité balnéaire naissante.

Le réseau d'influences ne s'arrête pas autour de Leroy. En prolongement de ce qui avait pu se passer avant la Première Guerre mondiale, le monde du journalisme prendra une large part à cette promotion. Maurice Martin, lui-même journaliste, partie prenante dans le concours de circonstances ayant amené Hossegor dans sa phase spéculative, n'est pas pour peu dans cette abondante couverture journalistique. De même, quelqu'un comme Hervé Lauwick, journaliste influent aux fortes attaches hossegoriennes, participe également dans les années trente à cette promotion médiatique. 


\section{Réseaux de clientèle}

évoquons pour finir une dernière acception de cette notion de réseau : le réseau de la clientèle qui fait bâtir à Hossegor et celui de ses architectes. Les origines géographiques des propriétaires hossegoriens obéissent à une logique relativement simple. Elles sont commandées, bien sûr, par des questions de proximité : le littoral aquitain est dès cette époque et encore maintenant le lieu de villégiature naturel des habitants des départements "continentaux» des régions Midi-Pyrénées et Aquitaine. Ces origines sont aussi conditionnées par des données historiques: la personnalité d'Eluère, ses relations, ses réseaux de publicité, expliquent la forte proportion de clientèle parisienne. Mais Eluère et Meunier-Godin utilisent également les réseaux locaux. Ils s'appuient, pour compléter ce contingent, sur une importante agence de Capbreton, la station voisine, l'agence Durand - laquelle s'installera à Hossegor un peu plus tard, vers 1934 - qui amène sur la nouvelle station sa clientèle traditionnelle, localisée principalement à Pau, Bordeaux et surtout Toulouse, les trois principales villes d'origine, après Paris, des propriétaires hossegoriens.

Il faut compter par la suite avec le bouche à oreille, les amis des clients qui deviennent aussi clients (on a tous en mémoire tel ou tel exemple de « colonisation » de ce genre : je connais pour ma part une vallée des Hautes-Pyrénées devenue villégiature charentaise en moins d'une génération, à partir de la fin des années cinquante).

\section{Réseaux d'architectes}

En ce qui concerne (dans le cas des constructions individuelles) le choix de l'architecte par le propriétaire, la notion de réseau est également déterminante, car pour un certain nombre d'entre eux, leur présence à Hossegor vient de la coïncidence entre leur origine géographique et celle des maitres d'ouvrage. Même si le cas de l'architecte implanté dans la région (au sens large) reste majoritaire, les Toulousains, Bordelais, Palois ou Parisiens venant en grand nombre faire construire à Hossegor entraîneront tout naturellement dans leur sillage un pourcentage non négligeable d'architectes issus des mêmes contrées. Et cela quand bien même l'on vient de loin, comme Léon Pfister, médecin à Saverne, dans le Bas-Rhin, qui fait intervenir les architectes strasbourgeois E. et J. Schwab pour la villa Irrintzina (1935), laquelle n'en sera pas moins construite en néo-basque, comme la plupart des œuvres hossegoriennes des architectes «importés ". L'architecte Jean Caresse, d'Orthez, est tout naturellement choisi par les Pouyanne, grande famille orthézienne, pour la construction de leur Villa d'Albret (1930). Mais il avait peu de temps auparavant (1929) achevé Cigalette pour un Bordelais. Serait-ce alors, inversement, plutôt Caresse qui aurait incité les Pouyanne à investir à Hossegor ? Rien n'est simple ni univoque. Pour preuve le cas de Valès, très probablement Toulousain, mais qui ne vient pas, lui, dans les «bagages » de quelque compatriote aucune de ses huit constructions recensées n'est en effet réalisée pour un Toulousain et qui semble plutôt venu par le canal de l'entreprise "La Toulousaine", particulièrement active sur Hossegor.

En conclusion de ce bref article, on pourrait rappeler ce que l'on a déjà suggéré : il y a ce qui est dit, ce qui est vu, ce qui est su; et puis il y a l'invisible, l'indicible, le souterrain, pour ne pas dire l'occulte. On comprend que c'est bien sûr ce deuxième aspect des choses qui est important, que c'est là où se passent vraiment les choses, 
même si cela a toutes les chances d'échapper à l'historien - a fortiori à Hossegor où, loin de toute planification systématique, c'est le pragmatisme qui a eu droit de cité. Le pragmatisme et l'intuition, le flair, qui est aussi l'art de savoir infléchir son programme en fonction de l'air du temps. Or, cela rejoint les constatations que l'on vient de faire sur l'architecture à Hossegor à cette époque, notamment sur la prééminence de l'expression néo-régionale. En effet, on est bien face à un cadre réglementaire, principalement sous la forme de cahiers des charges des lotissements. Mais ce cadre est réduit au strict minimum et ne prétend pas imposer une expression architecturale précise. On comprend donc que si la station a l'aspect homogène qu'elle $a$, si le régionalisme basco-landais en a été à ce point la règle tacite, ce n'est pas à ce cadre réglementaire vraiment réduit qu'elle le doit, mais à autre chose, de sûrement plus efficace. Plus que sur la contrainte, les hommes comme Eluère, à cette époque d'entrepreneurs, jouent principalement sur la force de conviction : convaincre, tout est là. Et dans cette démarche de persuasion, il est logique et naturel que la notion de réseau prenne toute sa place!

\section{NOTES}

1. Pour un historique plus complet de même que pour les références précises des sources sollicitées dans cet article, voir France. Inventaire général des monuments et des richesses artistiques de la France. Service régional d'Aquitaine. Hossegor, 1923-1939 : architecture et identité régionale. Réd. Claude Laroche. Bordeaux: APIA, Le Festin, 1993. (coll. Cahiers du patrimoine, $n^{\circ} 32$ ). De nombreux éléments de cet exposé sont également empruntés aux travaux de Gérard Maignan, notamment: Maxime Leroy à Hossegor. Soorts-Hossegor ; bulletin municipal d'informations, juillet 1999, $\mathrm{n}^{\circ} 36$; Maurice Martin à Hossegor. Soorts-Hossegor ; bulletin municipal d'informations, janvier 2000, $\mathrm{n}^{\circ} 38$; Paul Grunebaum-Ballin à Hossegor. Soorts-Hossegor; bulletin municipal d'informations, avril 2000, $\mathrm{n}^{\circ} 39$; Alfred Eluère à Hossegor. Soorts-Hossegor ; bulletin municipal d'informations, octobre 2000, $\mathrm{n}^{\circ} 41$; Hossegor et le courant d'Huchet. Soorts-Hossegor; bulletin municipal d'informations, juillet 2002, $n^{\circ} 48$.

2. Voir l'introduction de ce numéro par Bernard Toulier. Les réseaux de la villégiature en France.

3. Voir dans ce numéro l'article de Sophie Cueille. Les stratégies des investisseurs, des bords de ville aux bords de mer.

4. Fau, Guillaume. «Au sud-ouest d'éden : les Landes de Gaston Chérau ». Le Festin, juin 2003, n 46. P. 76-83, ici p. 79.

5. Rouillard, Dominique. Le site balnéaire. Liège-Bruxelles : Mardaga, 1984.

6. Voir note 2.

7. Drouin, Jean-Claude. Maxime Leroy (1873-1957) et le groupe d'Hossegor. Cahiers de l'université de Pau, 1982, nº 16 (ancienne série), actes de colloque (1985/05/22-23: Pau). P. 221-237, ici p. 231. 


\section{RÉSUMÉS}

La station balnéaire d'Hossegor (Landes) a connu son véritable lancement à partir de 1923, alors que le site avait été révélé quelques années auparavant, au tout début du siècle, par les séjours d'un groupe d'écrivains, parmi lesquels J.-H. Rosny jeune (1859-1948), Maxime Leroy (1873-1957) et Paul Margueritte (1860-1918). A partir de cet exemple landais, l'exposé tend à démontrer combien la création d'une station balnéaire, de sa découverte à sa promotion en passant par sa révélation au monde extérieur, doit au jeu des réseaux de tous ordres. Contemplatifs, les premiers occupants d'Hossegor n'auront pourtant de cesse de faire connaître le lieu en s'appuyant pour cela sur un puissant réseau de relations, notamment journalistiques. Actif, le promoteur de la station, Alfred Eluère (1893-1985), ne pourra assurer son succès qu'en utilisant le cercle relationnel très ouvert et très efficace que ses multiples activités - immobilières, mais aussi sportives et politiques - l'auront amené à constituer. Sa venue à Hossegor n'est d'ailleurs rien d'autre que le résultat de circonstances permises par le jeu de tout un tissu de relations au milieu duquel on trouve entre autres Maurice Martin (1861-1941), personnage haut en couleur, journaliste cultivant lui aussi au plus haut point le réseau des connaissances. Une fois la station lancée, la promotion immobilière saura jouer de tous les ressorts offerts par ces différents réseaux pour amener sur place la clientèle: de la propagande journalistique au réseau des agences immobilières en passant par le propre réseau relationnel d'Eluère. Parallèlement, le réseau des architectes sera lui aussi particulièrement actif, que ce soit pour s'attirer la commande ou pour propager la formule régionaliste. En conclusion, à côté de l'histoire officielle des stations balnéaires - une histoire au premier degré que les archives documentent relativement facilement - on ne peut passer à côté d'une autre histoire, plus souterraine mais qui a largement autant d'influence : celle des réseaux.

\section{INDEX}

Mots-clés : Abel Guichemerre, Aimé Meunier-Godin, Alexandre Darracq, Alfred Eluère, Bachet, Charles Derennes, Charles Siclis, Darracq, E. et J. Schwab, front de mer (à Hossegor), Gabriele d'Annunzio, golf, Henry Martinet, Hossegor, J.-H. Rosny jeune, Jean Caresse (architecte), Justin Boex (dit J.-H. Rosny jeune), Lauwick, Léon Pfister, lotissement du Rayol (Var), Louis Eluère, Marcel Gounouilhou, Maurice Martin, Maxime Leroy, Meunier-Godin, Paul Grunebaum-Ballin, Paul Margueritte, Pouyanne, Soorts-Hossegor, Sporting-Casino, Valès, Vigneau, villa Cigalette, villa d'Albret, villa Irrintzina, Côte d'Argent

\section{AUTEUR}

\section{CLAUDE LAROCHE}

Chercheur, service régional de l'Inventaire, Direction régionale des affaires culturelles d'Aquitaine. claude.laroche@culture.gouv.fr 